\section{Anemia e nível de hemoglobina como fatores prognósticos da mortalidade entre idosos residentes na comunidade: evidências da Coorte de Idosos de Bambuí, Minas Gerais, Brasil}

\author{
Anemia and hemoglobin level as prognostic factors \\ of mortality in community-dwelling elderly: \\ evidence from the Bambuí Cohort Study \\ on Aging, Minas Gerais State, Brazil
}

Clarice Lima Álvares da Silva 1 Maria Fernanda Lima-Costa ${ }^{2}$ Josélia Oliveira Araújo Firmo 2 Sérgio Viana Peixoto 2,3

\footnotetext{
${ }_{1}$ Centro de Pesquisas René Rachou, Fundação Oswaldo Cruz, Belo Horizonte, Brasil. 2 Núcleo de Estudos em Saúde Pública e Envelhecimento, Fundação Oswaldo Cruz/Universidade Federal de Minas Gerais, Belo Horizonte, Brasil. 3 Escola de Enfermagem, Universidade Federal de Minas Gerais, Belo Horizonte, Brasil

Correspondência C. L. A. Silva Centro de Pesquisas René Rachou, Fundação Oswaldo Cruz.

Rua Santa Maria 5 Conselheiro Lafaiete, $M G$ 36400-000, Brasil. claricelas@yahoo.com.br
}

\begin{abstract}
The aim of this study was to examine the influence of low hemoglobin ( $\mathrm{Hb}$ ) and anemia on mortality in the elderly in a ten-year followup (1997-2007). Among the 1,742 elderly participants in the baseline cohort from Bambui, Minas Gerais State, Brazil, 1,322 (82.3\%) were followed for an average of 8.9 years. Probability of survival was estimated by Kaplan-Meier and Cox's proportional hazard ratios (HR), and confidence intervals (95\%CI) were used, considering potential confounders. Anemia and low $\mathrm{Hb}$ (lowest tertile) were associated with increased risk of death $(H R=2.63,95 \% C I: 1.83-3.76$ and $H R=1.38,95 \% C I: 1.07-1.79$, respectively). The results show that anemia or low $\mathrm{Hb}$ (regardless of a diagnosis of anemia) should be interpreted and managed as independent determinants of mortality in this elderly population.
\end{abstract}

Anemia; Hemoglobins; Survivorship (Public Health); Aging

\section{Resumo}

O objetivo deste trabalho foi examinar a influência de baixos níveis de hemoglobina ( $\mathrm{Hb}) e$ anemia na mortalidade entre idosos ao longo de dez anos de seguimento (1997 a 2007). Entre os 1.742 idosos participantes da linha de base da coorte de Bambuí, Minas Gerais, Brasil, 1.322 (82,3\%) foram acompanhados pelo tempo médio de 8,9 anos. A probabilidade de sobrevida foi estimada pelo método de Kaplan-Meier e o modelo de riscos proporcionais de Cox foi utilizado, estimando-se os hazard ratios (HR) e intervalos de 95\% de confiança (IC95\%), considerando-se os potenciais fatores de confusão. Idosos anêmicos e com baixos níveis de $\mathrm{Hb}$ (primeiro tercil) apresentaram maior risco de óbito $(H R=2,63$; IC95\%: 1,83-3,76 e HR = 1,38; IC95\%: 1,07-1,79, respectivamente) comparados aos não anêmicos e aos de maior nível de Hb. Os resultados demonstram que a ocorrência de anemia elou baixos níveis de $\mathrm{Hb}$ (independente do diagnóstico de anemia) devem ser interpretados e tratados como determinante independente da mortalidade nessa população idosa.

Anemia; Hemoglobinas; Sobrevida; Envelhecimento 


\section{Introdução}

Anemia é uma condição comum entre idosos, com prevalência elevando-se após os 50 anos e alcançando cerca de $20 \%$ da população com 85 anos ou mais de idade $1,2,3,4$, sendo por muito tempo considerada uma condição resultante do próprio processo de envelhecimento. Entretanto, estudos mais recentes mostraram que a anemia em idosos é um fator prognóstico independentemente de piores condições de saúde 2,5,6,7,8, aumentando ainda mais a vulnerabilidade para suas complicações 5,9,10,11.

Estudos de base populacional entre idosos para determinar a prevalência de anemia e os fatores associados ao nível de hemoglobina $(\mathrm{Hb})$ ainda são escassos 12. No Brasil, três estudos realizados em populações idosas selecionadas 13,14,15 e um estudo de base populacional 16 mostraram prevalências de anemia variando entre $4 \%$ e $12 \%$. Na linha de base da coorte de base populacional de idosos de Bambuí, Minas Gerais, Brasil, a prevalência da anemia foi de 4,5\%. Nesse estudo, verificou-se que baixos níveis de $\mathrm{Hb}$ estavam associados ao pior estado nutricional e à maior utilização de serviços de saúde, mesmo após ajustamentos por idade, escolaridade, tabagismo, diabetes, hipertensão, artrite, níveis séricos de creatinina e número de medicamentos consumidos 17 .

Estudos conduzidos em populações idosas nos Estados Unidos, Canadá e em alguns países europeus evidenciaram maior risco de morte entre aqueles com anemia, independentemente de outros fatores relevantes 5,9,18,19,20,21,22. Além disso, baixos níveis de $\mathrm{Hb}$, independente do diagnóstico de anemia, também apresentaram associação significativa com mortalidade em algumas populações idosas 18,19,21,23. Esses resultados são reforçados por estudos que mostraram maior risco de morte entre idosos com redução nos níveis de $\mathrm{Hb}$ ou anemia incidente nos primeiros anos de seguimento 8,18 . No entanto, o impacto da anemia e dos baixos níveis de $\mathrm{Hb}$ na sobrevida de populações idosas residentes em países em desenvolvimento, inclusive o Brasil, ainda não foi avaliado.

No presente trabalho, foram utilizados dados da coorte de base populacional de idosos em Bambuí 24 para examinar a influência dos níveis de $\mathrm{Hb}$, assim como da presença de anemia na mortalidade ao longo de dez anos entre idosos residentes na comunidade.

\section{Material e métodos}

\section{Coorte de idosos de Bambuí}

A Coorte de Idosos de Bambuí é conduzida na cidade de mesmo nome (aproximadamente 15 mil habitantes), situada no Estado de Minas Gerais. O município foi uma região endêmica para Doença de Chagas, infecção causada pelo protozoário Trypanosoma cruzi. A transmissão da infecção foi interrompida na década de 70 , mas a prevalência de sorologia positiva permanece alta na população idosa devido ao efeito de coorte. As principais causas de morte entre idosos residentes em Bambuí são acidente vascular encefálico (AVE), doença de Chagas e doença isquêmica coronariana (taxas de mortalidade 110,61 e 42/100 mil habitantes, respectivamente) 24,25 .

O delineamento da coorte e os procedimentos adotados foram descritos em publicações anteriores $24,25,26$. Resumidamente, os participantes da linha de base foram identificados por meio de um censo completo realizado na cidade em 1996. Todos os 1.742 residentes na área urbana do município com 60 ou mais anos de idade em $1^{\circ} \mathrm{de}$ janeiro de 1997 foram convidados a participar do estudo de coorte. A linha de base do estudo foi estabelecida entre fevereiro e maio de 1997, incluindo a realização de entrevistas, exame físico e coleta de sangue. Os participantes entrevistados e examinados na linha de base da coorte foram semelhantes à população idosa residente na cidade em relação à idade, sexo, estado civil, escolaridade e renda ${ }^{25}$. Estes são submetidos a visitas anuais de acompanhamento, que consistem de entrevista e verificação da ocorrência de óbitos. O Projeto Bambuí foi aprovado pelo Comitê de Ética da Fundação Oswaldo Cruz, Rio de Janeiro, e todos os participantes assinaram um termo de consentimento livre e esclarecido.

\section{Mortalidade}

Óbitos ocorridos entre o estabelecimento da linha de base (1997) e 31 de dezembro de 2007 foram incluídos na presente análise. Os óbitos foram relatados por familiares durante entrevistas realizadas nos seguimentos anuais e confirmados pelo Sistema de Informações sobre Mortalidade (SIM). Certidões de óbito foram obtidas para $98,9 \%$ dos idosos. Na presente análise, foram considerados os óbitos por todas as causas como variável principal.

\section{Variáveis da linha de base}

Para a presente análise, foram consideradas as seguintes informações da linha de base da co- 
orte: nível de Hb e diagnóstico de anemia, sexo, idade, escolaridade, cor da pele, tabagismo atual, consumo de álcool nos últimos 12 meses, índice de massa corporal (IMC), infecção pelo T. cruzi, pressão arterial sistólica, creatinina sérica, diabetes mellitus, história de AVE, presença de angina, relato de infarto, percepção da própria saúde e função cognitiva.

A dosagem do nível de $\mathrm{Hb}$ foi realizada utilizando-se contador eletrônico (Coulter Count T-890, Estados Unidos). Esse nível foi distribuído em tercis, além de ser considerado também o diagnóstico de anemia segundo o critério da Organização Mundial da Saúde (OMS) 27 (valores abaixo de 13g/dL para o sexo masculino e $12 \mathrm{~g} / \mathrm{dL}$ para feminino).

Foi entendido como tabagista atual o participante que relatou ter fumado no mínimo 100 cigarros durante a vida e continuava fumando no momento da entrevista. O uso de bebidas alcoólicas foi avaliado pelo consumo, em qualquer quantidade, nos doze meses anteriores à entrevista. O IMC foi calculado a partir da razão entre o peso e o quadrado da altura $\left(\mathrm{kg} / \mathrm{m}^{2}\right)^{28}$. A avaliação antropométrica foi realizada com uso de técnicas e equipamentos padrões (CMS Weighing Equipment Ltd., Londres, Reino Unido) e com os participantes vestindo roupas leves e sem sapatos.

A infecção por T. cruzi foi avaliada por três testes realizados simultaneamente, sendo um ensaio de hemaglutinação (Biolab Mérieux, Rio de Janeiro, Brasil) e dois testes ELISA (Enzyme-Linked Immunonoabsorbent Assay) (Abbott Laboratories, Estados Unidos e Wiener Laboratories, Argentina). A infecção foi definida pela sorologia positiva em todos os três exames e a ausência de infecção quando todos os resultados foram negativos. A pressão arterial sistólica foi definida como a média das duas últimas medidas, entre as três coletadas, utilizando-se protocolo padronizado. A dosagem sérica de creatinina foi realizada por método enzimático em equipamento padronizado (Eclipse Vitalab; Merck, Holanda). O diagnóstico de diabetes mellitus foi definido pela dosagem de glicemia maior ou igual a $126 \mathrm{mg} / \mathrm{dL}$ e/ou o uso de hipoglicemiante oral ou insulina. A história de AVE 29 e a presença de angina 30 foram verificadas pela utilização de instrumentos padronizados. Foi considerada também a história de diagnóstico médico para infarto. A percepção da própria saúde foi verificada pela resposta à questão “Como você avalia sua própria saúde?", sendo considerada na análise a resposta ruim, agrupando-se as demais (muito boa, boa e regular). A função cognitiva foi avaliada pelo uso da versão em português do Mini-Mental State Examination (MMSE), sendo essa variável incluída na análise como contínua 31.
Todos os procedimentos foram realizados por entrevistadores e técnicos devidamente treinados e certificados. As entrevistas foram realizadas na residência do participante e respondidas pelo próprio idoso, exceto quando ele estava impossibilitado por déficit cognitivo ou algum problema de saúde, utilizando-se, nesse caso, um respondente próximo $(4,8 \%$; $\mathrm{n}=69)$. As medidas de pressão arterial, antropometria e dosagens bioquímicas foram realizadas na clínica de campo do projeto, salvo na impossibilidade de o participante se locomover até a clínica. Nesse caso, as medidas foram realizadas no domicílio. A coleta de sangue foi feita após recomendação de jejum de 12 horas. Maiores detalhes podem ser vistos em publicações anteriores $24,25,26$.

\section{Análise dos dados}

Foram utilizados o teste do qui-quadrado de Pearson para comparação de frequências, teste t de Student para comparação de médias entre anêmicos e não anêmicos e análise de variância complementada com teste de Bonferroni para comparação de médias entre os tercis de Hb.

O cálculo das probabilidades não ajustadas de sobrevida foi realizado pelo método de Kaplan-Meier, segundo o diagnóstico de anemia e os tercis de $\mathrm{Hb}$.

A análise multivariada da associação entre diagnóstico de anemia e tercis de $\mathrm{Hb}$ com mortalidade ao longo do período de seguimento da coorte foi baseada em estimativas do $h a-$ zard ratio (HR) e respectivos intervalos de $95 \%$ de confiança (IC95\%), utilizando o modelo de riscos proporcionais de Cox, tendo sido confirmada a premissa da proporcionalidade dos riscos ao longo do tempo. Tal análise foi realizada pela construção de quatro modelos para anemia e tercis de $\mathrm{Hb}$, com a inclusão progressiva de potenciais fatores de confusão para mortalidade entre idosos. No primeiro modelo (M1), as estimativas não foram ajustadas por fatores de confusão. O segundo modelo (M2) foi ajustado por idade, sexo, escolaridade e cor da pele. No terceiro modelo (M3), foram incluídas as variáveis tabagismo atual e relato de consumo de álcool. No último modelo (M4), foram incluídas as variáveis de condição de saúde do idoso (IMC, níveis séricos de creatinina, sorologia para $T$. cruzi, pressão arterial sistólica, história de AVE, presença de angina, relato de infarto, diabetes mellitus, percepção da própria saúde e escore do MMSE). As variáveis utilizadas nos modelos foram consideradas por apresentarem associação com o nível de Hb e por serem consideradas preditoras de mortalidade na Coorte de Idosos de Bambuí 19,20,32,33,34,35. 
Todas as análises foram realizadas com utilização do pacote estatístico Stata 10.0 (Stata Corp., College Station, Estados Unidos).

\section{Resultados}

Entre os 1.606 idosos participantes da linha de base do estudo, 1.322 (82,3\%) com informações para todas as variáveis consideradas foram incluídos na presente análise. Durante o tempo médio de seguimento de 8,9 anos (11.814,7 pessoas-ano sob risco), 475 óbitos foram registrados e $82(6,2 \%)$ idosos foram perdidos. Entre os idosos incluídos no presente estudo, 503 (38,1\%) eram homens e 819 (61,9\%) eram mulheres. O nível médio de $\mathrm{Hb}$ nessa população foi igual a 14,5 g/dL (desvio-padrão $=1,4$ ) e a prevalência de anemia foi de $3,8 \%$ $(\mathrm{n}=50)$, sendo $4,6 \%(\mathrm{n}=23)$ no sexo masculino e $3,3 \%(n=27)$ no feminino ( $p=0,238)$.

As Tabelas 1 e 2 apresentam o perfil da coorte para variáveis sociodemográficas, condições de saúde, estado nutricional e hábitos de vida segundo diagnóstico de anemia e tercis de $\mathrm{Hb}$, respectivamente. Idosos anêmicos apresentaram significativamente menor IMC, maiores valores médios de pressão sistólica e creatinina sérica (Tabela 1). Quando a população foi estratificada por tercis do nível de $\mathrm{Hb}$, maiores valores para essa variável foram encontrados entre os idosos mais jovens, do sexo masculino, fumantes, com relato de consumo de álcool, com menores escores do MMSE e maiores valores para creatinina sérica, além de menor frequência de história de AVE, angina e de infecção pelo T. cruzi (Tabela 2).

As Figuras 1 e 2 mostram a probabilidade de sobrevida durante os dez anos de seguimento, segundo o diagnóstico de anemia e tercis de $\mathrm{Hb}$, respectivamente. Os gráficos ilustram a menor probabilidade de sobrevida tanto entre idosos anêmicos quanto entre aqueles no tercil inferior do nível de $\mathrm{Hb}$.

As taxas de mortalidade e a associação entre anemia e nível de $\mathrm{Hb}$ com a mortalidade em 10 anos estão apresentadas na Tabela 3. Os 475 óbitos (210 homens e 265 mulheres) ocorridos nos 10 anos de seguimento representaram 35,9\% da população idosa incluída nessa análise. Taxas de mortalidade entre idosos com e sem anemia, segundo critério da OMS, foram 95,7 e 38,5/1.000 pessoas-ano, respectivamente. A mortalidade foi maior no tercil basal (44,5/1.000 pessoas-ano), diminuindo no segundo e terceiro tercis (36,2 e 39,8/1.000 pessoas-ano, respectivamente).

Considerando o diagnóstico de anemia, todos os quatro modelos construídos evidenciaram maior risco de morte entre idosos anêmicos. Quando ajustado por todos os potenciais fatores

Características dos participantes do estudo, segundo diagnóstico de anemia. Linha de base da Coorte de Idosos de Bambuí, Minas Gerais, Brasil.

\begin{tabular}{|c|c|c|c|c|}
\hline \multirow[t]{2}{*}{ Variáveis } & \multirow[t]{2}{*}{ Total } & \multicolumn{2}{|c|}{ Anemia } & \multirow[t]{2}{*}{ Valor de $\mathrm{p}^{1}$} \\
\hline & & Sim & Não & \\
\hline Idade em anos [média (desvio-padrão)] & $68,8(6,9)$ & $70,6(7,4)$ & $68,7(6,9)$ & 0,051 \\
\hline Sexo masculino [n (\%)] & $503(38,1)$ & $23(46,0)$ & $480(37,7)$ & 0,238 \\
\hline Escolaridade $<4$ anos [n (\%)] & $837(63,3)$ & $35(70,0)$ & $802(63,1)$ & 0,317 \\
\hline Cor da pele não branca [n (\%)] & $528(39,9)$ & $17(34,0)$ & $511(40,2)$ & 0,382 \\
\hline Tabagismo atual [n (\%)] & $226(17,1)$ & $6(12,0)$ & $220(17,3)$ & 0,329 \\
\hline Consumo de álcool nos últimos 12 meses [n (\%)] & $278(21,0)$ & $9(18,0)$ & $269(21,2)$ & 0,590 \\
\hline Índice de massa corporal em kg/m² [média (desvio-padrão)] & $25,2(5,0)$ & $23,7(4,0)$ & $25,2(5,0)$ & 0,039 \\
\hline Pressão arterial sistólica em mmHg [média (desvio-padrão)] & $137,2(22,6)$ & $147,3(24,6)$ & $136,8(22,4)$ & 0,001 \\
\hline Creatinina sérica em mg/dL [média (desvio-padrão)] & $0,9(0,3)$ & $1,2(1,1)$ & $0,9(0,2)$ & $<0,001$ \\
\hline Escore no Mini-MentalStateExamination [média (desvio-padrão)] & $24,9(4,1)$ & $25,1(3,6)$ & $24,9(4,1)$ & 0,729 \\
\hline Diabetes mellitus [n (\%)] & $195(14,8)$ & $9(18,0)$ & $186(14,6)$ & 0,509 \\
\hline História de acidente vascular encefálico [n (\%)] & $44(3,3)$ & $1(2,0)$ & $43(3,4)$ & 0,593 \\
\hline Presença de angina [n (\%)] & $123(9,3)$ & $7(14,0)$ & $116(9,1)$ & 0,246 \\
\hline Relato de infarto [n (\%)] & $61(4,6)$ & $4(8,0)$ & $57(4,5)$ & 0,248 \\
\hline Infecção por Trypanosoma cruzi [n (\%)] & $494(37,4)$ & $13(26,0)$ & $481(37,8)$ & 0,090 \\
\hline Percepção ruim da própria saúde [n (\%)] & $332(25,1)$ & $8(16,0)$ & $324(25,5)$ & 0,130 \\
\hline
\end{tabular}

* Teste t de Student para comparações entre médias e teste do qui-quadrado de Pearson para comparações entre proporções. 
Características dos participantes do estudo, segundo os níveis de hemoglobina em tercis. Linha de base da Coorte de Idosos de Bambuí, Minas Gerais, Brasil.

\begin{tabular}{|c|c|c|c|c|}
\hline \multirow[t]{2}{*}{ Variáveis } & \multicolumn{3}{|c|}{ Nível de hemoglobina * } & \multirow[t]{2}{*}{ Valor de $p$ ** } \\
\hline & Tercil 1 & Tercil 2 & Tercil 3 & \\
\hline Idade em anos [média (desvio-padrão)] & $69,5(7,1)$ a & $68,8(7,0)$ ab & $67,8(6,6) b$ & 0,002 \\
\hline Sexo masculino $[\mathrm{n}(\%)]$ & $67(14,3)$ & $134(30,3)$ & $302(73,7)$ & $<0,001$ \\
\hline Escolaridade $<4$ anos [n (\%)] & $301(64,2)$ & $285(64,3)$ & $251(61,2)$ & 0,570 \\
\hline Cor da pele não branca [n (\%)] & $198(42,2)$ & $174(39,3)$ & $156(38,1)$ & 0,426 \\
\hline Tabagismo atual $[\mathrm{n}(\%)]$ & $49(10,5)$ & $75(16,9)$ & $102(24,9)$ & $<0,001$ \\
\hline Consumo de álcool nos últimos 12 meses [n (\%)] & $52(11,1)$ & $90(20,3)$ & $136(33,3)$ & $<0,001$ \\
\hline Índice de massa corporal em kg/m² [média (desvio-padrão)] & $25,1(4,7)$ & $25,3(5,3)$ & $25,1(4,9)$ & 0,756 \\
\hline Pressão arterial sistólica em mmHg [média (desvio-padrão)] & $137,7(23,0)$ & $135,4(22,1)$ & $138,5(22,5)$ & 0,119 \\
\hline Creatinina sérica em mg/dL [média (desvio-padrão)] & $0,9(0,4)$ a & $0,9(0,2)$ a & $1,0(0,2) b$ & $<0,001$ \\
\hline Diabetes mellitus [n (\%)] & $57(12,2)$ & $66(14,9)$ & $72(17,6)$ & 0,078 \\
\hline História de acidente vascular encefálico [n (\%)] & $15(3,2)$ & $22(5,0)$ & $7(1,7)$ & 0,029 \\
\hline Presença de angina [n (\%)] & $56(12,0)$ & $42(9,5)$ & $25(6,1)$ & 0,011 \\
\hline Relato de infarto [n (\%)] & $23(4,9)$ & $19(4,3)$ & $19(4,7)$ & 0,910 \\
\hline Infecção por Trypanosoma cruzi [n (\%)] & $194(41,4)$ & $170(38,4)$ & $130(31,7)$ & 0,011 \\
\hline Percepção ruim da própria saúde [n (\%)] & $132(28,1)$ & $106(23,9)$ & $94(22,9)$ & 0,160 \\
\hline
\end{tabular}

* Tercil 1: 8,4-13,9g;/dL; tercil 2: 14,0 a 15,0 g/dL; tercil 3: 15,1 a 19,5 g/dL;

** Análise de variância e teste de Bonferroni para comparações entre médias e teste do qui-quadrado de Pearson para comparações entre proporções. Nota: as letras representam diferenças estatísticas no teste ANOVA/Bonferroni.

Figura 1

Probabilidade de sobrevida em dez anos, segundo a presença de anemia na linha de base. Coorte de Idosos de Bambuí, Minas Gerais, Brasil, 1997-2007.

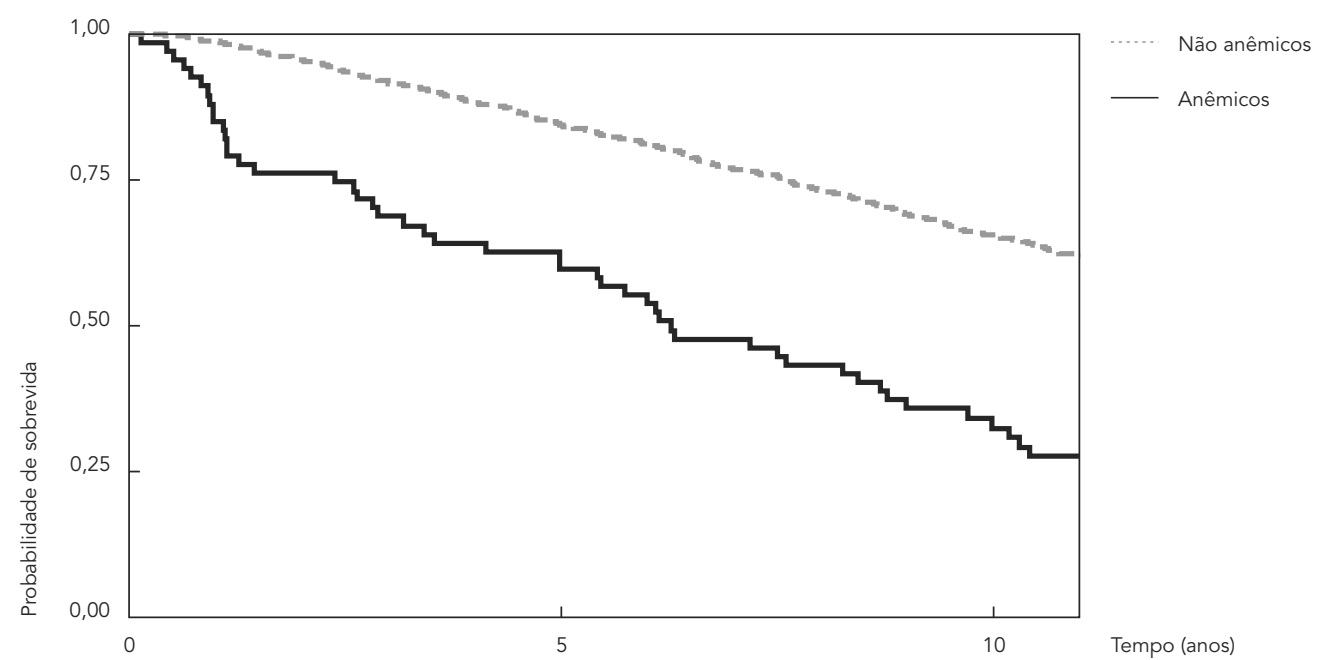


Probabilidade de sobrevida em dez anos, segundo tercis de hemoglobina na linha de base, Coorte de Idosos de Bambuí, Minas Gerais, Brasil, 1997-2007.

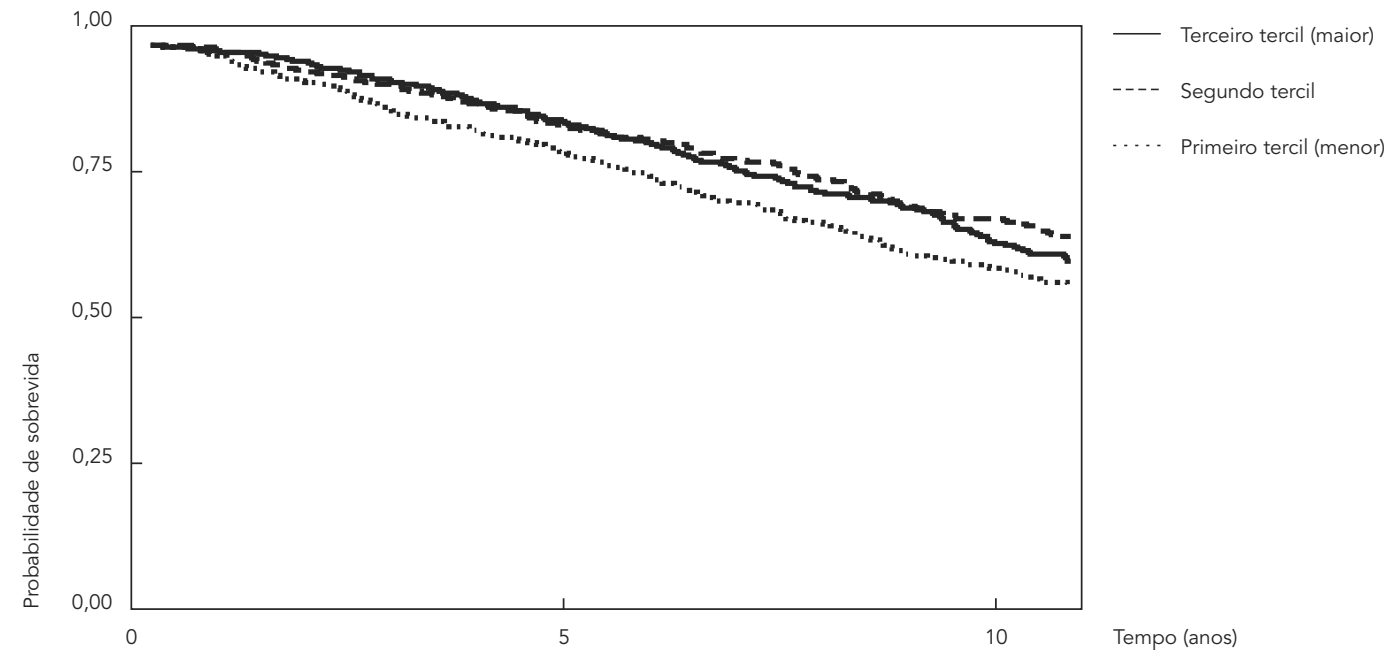

Tabela 3

Taxas de mortalidade e hazard ratios (HR) em dez anos de seguimento, segundo diagnóstico de anemia e nível de hemoglobina em tercis avaliados na linha de base. Coorte de Idosos de Bambuí, Minas Gerais, Brasil, 1997-2007.

\begin{tabular}{|c|c|c|c|c|c|c|c|c|c|}
\hline \multirow[t]{2}{*}{ Condição } & \multirow{2}{*}{$\begin{array}{c}\text { Taxas de } \\
\text { mortalidade por } \\
1.000 \text { pessoas/ano }\end{array}$} & \multicolumn{2}{|c|}{ Modelo 1} & \multicolumn{2}{|c|}{ Modelo 2} & \multicolumn{2}{|c|}{ Modelo 3} & \multicolumn{2}{|c|}{ Modelo 4} \\
\hline & & HR & IC95\% & HR & IC95\% & HR & IC95\% & HR & IC95\% \\
\hline \multicolumn{10}{|l|}{ Anemia } \\
\hline Não & 38,5 & 1,00 & - & 1,00 & - & 1,00 & - & 1,00 & - \\
\hline Sim & 95,7 & 2,61 & $1,83-3,71$ & 2,52 & $1,76-3,58$ & 2,60 & $1,82-3,72$ & 2,23 & $1,52-3,26$ \\
\hline \multicolumn{10}{|c|}{ Níveis de hemoglobina } \\
\hline Tercil 3 & 39,8 & 1,00 & - & 1,00 & - & 1,00 & - & 1,00 & - \\
\hline Tercil 2 & 36,2 & 0,90 & $0,72-1,14$ & 1,04 & $0,81-1,34$ & 1,04 & $0,81-1,34$ & 1,09 & $0,85-1,40$ \\
\hline Tercil 1 & 44,5 & 1,12 & $0,90-1,40$ & 1,34 & $1,03-1,73$ & 1,39 & $1,07-1,80$ & 1,36 & $1,05-1,75$ \\
\hline
\end{tabular}

IC95\%: intervalo de 95\% de confiança.

Modelo 1: bruto; Modelo 2: ajustado por idade, sexo, escolaridade e cor da pele; Modelo 3: ajustado pelas variáveis do Modelo 2, além de tabagismo e consumo de álcool; Modelo 4: ajustado pelas variáveis do Modelo 3, além de índice de massa corporal, pressão arterial sistólica, creatinina sérica,

Escore no Mini-Mental State Examination, diabetes mellitus, história de acidente vascular encefálico, presença de angina, relato de infarto,

de confusão, o diagnóstico de anemia apresentou um HR = 2,23 (IC95\%: 1,52-3,26). Estratificando a população em tercis de $\mathrm{Hb}$, a análise não ajustada não mostrou associação significativa com mortalidade, tanto no nível mais baixo (HR = 1,12; IC95\%: 0,90-1,40), quanto no nível intermediário (HR = 0,90; IC95\%: 0,72-1,14), com- parado ao terceiro tercil. Quando foi realizado o ajuste pelos fatores de confusão, em todos os modelos foi observada associação significativa entre o tercil inferior e a mortalidade por todas as causas. Considerando o modelo ajustado por todas as variáveis pesquisadas, os idosos do tercil inferior apresentaram maior risco de óbito 
(HR = 1,36; IC95\%: 1,05-1,75), quando comparados aos de maior nível de $\mathrm{Hb}$ (Tabela 3).

\section{Discussão}

Os resultados deste trabalho indicam que tanto baixos níveis da $\mathrm{Hb}$, quanto a presença de anemia estão associados ao aumento do risco para mortalidade entre idosos. Essas associações persistiram mesmo após ajustamentos por diversos fatores relevantes, incluindo a situação socioeconômica, comportamentos em saúde, percepção da própria saúde e marcadores biológicos das condições de saúde.

A associação entre diagnóstico de anemia e mortalidade observada entre idosos residentes em Bambuí é consistente com o observado em populações de renda mais alta. Entre idosos participantes do Cardiovascular Healthy Study (CHS; Estados Unidos) 18, a anemia na linha de base foi associada ao maior risco de morte durante 11 anos de seguimento (HR=1,38; IC95\%: 1,19-1,59), mesmo após ajuste para fatores de confusão. A análise dos dados do Third National Health and Nutrition Study (NHANES III) 5, incluindo 7.171 americanos com 50 anos ou mais, acompanhados por 12 anos, mostrou que o risco de morte foi 1,6 vez maior entre anêmicos $(p<0,001)$, sendo essa associação mais forte para anemia moderada ou severa (risco $=2,4$; $\mathrm{p}<0,001$ ).

Dois outros importantes estudos norte-americanos desenvolvidos em populações idosas mostraram o impacto da anemia na sobrevida desse grupo. Entre os 1.701 idosos ( $\geq 65$ anos) participantes do Duke Established Populations for Epidemiologic Studies of the Elderly ${ }^{20}$, desenvolvido na Carolina do Norte, a anemia foi significativamente associada ao maior risco de morte durante 8 anos de seguimento ( $\mathrm{HR}=1,4$; IC95\%: 1,2-1,6), tendo em conta ajuste por fatores de confusão. Entre 3.607 idosos ( $\geq 71$ anos) participantes do Established Populations for Epidemiologic Studies of the Elderly (EPESE) 21, verificou-se que a anemia aumentou significativamente o risco de óbito por todas as causas (HR = 1,63; IC95\%: 1,37-1,95), sendo essa associação ainda mais forte quando os idosos com doenças e/ou condições clínicas importantes foram excluídos da análise (HR = 2,12; IC95\%: 1,48$3,04)$, reforçando a hipótese de que a anemia pode ser considerada um preditor independente de mortalidade nesse grupo.

Além dos estudos norte-americanos, resultados obtidos em outros países também demonstraram associação entre anemia e mortalidade. Entre idosos italianos (65 a 84 anos), essa condição aumentou 2,54 vezes (IC95\%: 2,14-3,03) o ris- co de morte após 3,5 anos de acompanhamento, em relação aos idosos não anêmicos 19. Estudo conduzido com 17.030 idosos ( $\geq 66$ anos) canadenses acompanhados por três anos demonstrou forte associação entre anemia e mortalidade (HR = 4,29; IC95\%: 3,55-5,12), considerando possíveis fatores de confusão ${ }^{9}$. Na Holanda, o acompanhamento de 562 idosos mais velhos (85+ anos) durante 5 anos também encontrou associação significativa entre anemia avaliada na linha de base e mortalidade geral $(\mathrm{HR}=1,41$; IC95\%: 1,13-1,76) 8.

Independentemente do diagnóstico de anemia, a análise do risco de mortalidade de acordo com os níveis de $\mathrm{Hb}$ (tercis de $\mathrm{Hb}$ ) revelou a ocorrência de uma relação dose-resposta para essa condição, sendo que o tercil basal de $\mathrm{Hb}$ (tercil 1) apresentou um risco de morte significativamente maior que o terceiro tercil entre os idosos residentes em Bambuí. Tais resultados foram reforçados quando se levou em conta o nível de Hb como variável contínua, que apresentou um $\mathrm{HR}=0,88$ (IC95\%: 0,81-0,94), após ajuste por todas as variáveis de confusão consideradas nesse estudo (dados não mostrados), indicando que o maior nível de hemoglobina se comporta como fator protetor da mortalidade nessa população.

De forma semelhante aos resultados encontrados em Bambuí, baixos níveis de $\mathrm{Hb}$ foram significativamente associados ao maior risco de morte por todas as causas entre idosos residentes nos Estados Unidos 18,21, na Itália 19 e no Canadá 9 . Chama atenção que em dois desses estudos foi observada uma associação em forma de J invertido entre os níveis de hemoglobina e a mortalidade geral 9,18, resultados que não foram reproduzidos na Coorte de Idosos de Bambuí (dados não mostrados). Entre idosos residentes na Carolina do Norte (Estados Unidos), uma maior sobrevida após 8 anos de acompanhamento ocorreu em mulheres com nível de Hb entre 13 e 14g/dL e em homens com nível de $\mathrm{Hb}$ entre 14g/dL e 15g/dL, ambos superiores ao recomendado pela OMS 20 .

Cabe ressaltar que a associação entre maiores níveis de $\mathrm{Hb}$ e proteção contra mortalidade em idosos de Bambuí corrobora com a ampla discussão acerca da adequação dos pontos de cortes propostos pela OMS para o diagnóstico de anemia entre idosos. Ficou demonstrado que idosos com níveis de $\mathrm{Hb}$ abaixo 13,9g/dL (primeiro tercil) na linha de base apresentaram risco de óbito $36 \%$ maior após 10 anos de seguimento, comparado ao terceiro tercil $(\mathrm{Hb}>15,0 \mathrm{~g} / \mathrm{dL})$. Esse achado vai de encontro aos resultados de uma revisão sistemática sobre o tema, que demonstrou que níveis de $\mathrm{Hb}$ iguais ou superiores a $14 \mathrm{~g} / \mathrm{dL}$ estão relacionados a melhores condições de saúde em tal grupo populacional 36 . 
Algumas questões metodológicas devem ser consideradas na interpretação dos resultados do presente estudo. Como todo estudo de coorte, a sobrevivência seletiva antes da entrada na coorte deve ser considerada, uma vez que idosos com baixos valores de hemoglobina apresentam maior risco de morte. Se presente, esse viés pode ter enfraquecido as associações encontradas. Em se tratando de um estudo observacional, a possibilidade de confusão residual não deve ser descartada. Todavia, destaca-se que os principais fatores que poderiam influenciar os níveis de $\mathrm{Hb}$ e a mortalidade dessa população idosa foram considerados na presente análise. Por fim, a etiologia da anemia não pôde ser determinada para esse grupo, não sendo possível avaliar a magnitude desse efeito na mortalidade dos idosos de Bambuí. Por outro lado, cabe destacar que os principais fatores de confusão considerados nesta análise foram obtidos por medidas diretas, por entrevistadores treinados e por técnicas padronizadas, o que aumenta a precisão dos dados coletados. Os óbitos ocorridos durante os dez anos de acompanhamento foram confirmados por consulta ao SIM, aumentando também a precisão da medida do desfecho do estudo. As perdas ocorridas durante o tempo de seguimento foram mínimas $(6,2 \%)$, reforçando a consistência interna dessa coorte. Além disso, esse é o primeiro estudo sobre o impacto da anemia e dos níveis de hemoglobina na mortalidade de uma população idosa residente em um país em desenvolvimento e foi possível retratar a associação já observada em outros países.

Em resumo, os resultados verificados na Coorte de Idosos de Bambuí mostraram que tanto a anemia quanto menores níveis de hemoglobina foram preditores independentes da mortalidade por todas as causas nessa população. Assim, a ocorrência de anemia e, em especial, de baixos níveis de $\mathrm{Hb}$ (independentemente do diagnóstico de anemia), possivelmente não deve ser considerada como simples marcador bioquímico de doenças ou achado fisiológico do processo de envelhecimento. Ao contrário, deve ser interpretada e tratada como determinante independente de morbidade, mortalidade e redução da qualidade de vida nesse grupo 6 .

\section{Resumen}

El objetivo de este trabajo fue evaluar la influencia de los bajos niveles de hemoglobina (Hb) y anemia sobre la mortalidad de ancianos a lo largo de diez años (19972007). De los 1.742 ancianos participantes de la cohorte de Bambuí, Minas Gerais, Brasil, a 1.322 (82,3\%) se les realizó un seguimiento promedio de 8,9 años. Se utilizó el método de Kaplan-Meier para estimar la probabilidad de supervivencia y el modelo de riesgos proporcionales de Cox para poder realizar una estimación del cociente de riesgo - hazard ratios $(H R)$ - con intervalos de confianza de un 95\%. Asimismo, fueron considerados factores potenciales de confusión. Ancianos anémi- cos y con niveles bajos de $\mathrm{Hb}$ (primer tercil) presentaron un riesgo superior de morir ( $H R=2,63$; IC95\%: 1,83 a 3,76 y HR = 1,38; IC95\%: 1,07 a 1,79, respectivamente), en comparación con los individuos no anémicos y con los que presentaron niveles superiores de Hb. Los resultados indican que la ocurrencia de anemia y/o bajos niveles de $\mathrm{Hb}$ (independiente del diagnóstico) se debe interpretar y manejar como factores determinantes independientes de la mortalidad entre una determinada población geriátrica..

Anemia; Hemoglobinas; Sobrevida; Envejecimiento 


\section{Colaboradores}

C. L. A. Silva realizou a análise dos dados, a interpretação dos resultados e a redação do manuscrito. M. F. Lima-Costa e S. V. Peixoto orientaram o trabalho, participando da discussão dos resultados, redação e revisão do texto final. J. O. A. Firmo coordenou o trabalho de campo de Bambuí e participou da discussão dos resultados e revisão do texto.

\section{Agradecimentos}

À Financiadora de Estudos e Projetos (processo no 6694009-00), ao CNPq (processo no 140923/2009-8).

\section{Referências}

1. Balducci L. Epidemiology of anemia in the elderly: information on diagnostic evaluation. J Am Geriatr Soc 2003; 51(3 Suppl):S2-9.

2. Guralnik JM, Eisenstaedt RS, Ferrucci L, Klein HG, Woodman RC. Prevalence of anemia in persons 65 years and older in the United States: evidence for a high rate of unexplained anemia. Blood 2004; 104:2263-8.

3. Lippi G, Franchini M, Salvagno GL, Montagnana M, Targher. Determinants of anemia in the very elderly: a major contribution from impaired renal function? Blood Transfus 2010; 8: 44-8.

4. Price EA, Mehra R, Holmes TH, Schrier SL. Anemia in older persons: etiology and evaluation. Blood Cells Mol Dis 2011; 46:159-65.

5. Shavelle RM, Mackenzie R, Paculdo DR. Anemia and mortality in older persons: does the type of anemia affect survival? Int J Hematol 2012; 95: 248-56.

6. Chaves PH, Xue QL, Guralnik JM, Ferrucci L, Volpato S, Fried LP. What constitutes normal hemoglobin concentration in community-dwelling disabled older women? J Am Geriatr Soc 2004; 52:1811-6.

7. Woodman R, Ferrucci L, Guralnik J. Anemia in older adults. Curr Opin Hematol 2005; 12:123-8.
8. den Elzen WPJ, Willens JM, Westendorp RGJ, Craen AJM, Assendelft WJJ, Gussekloo J. Effect of anemia and comorbity on functional status and mortality in old age: results from the Leiden 85-plus Study. CMAJ 2009; 181:151-7.

9. Culleton BF, Manns BJ, Zhang J, Tonelli M, Klarenbach S, Hemmelgan BR. Impact of anemia on hospitalization and mortality in older adults. Blood 2006; 107:3841-6.

10. Landi F, Russo A, Danese P, Liperoti R, Barillaro C, Bernabei R, et al. Anemia status, hemoglobin concentration and mortality in nursing home older residents. J Am Med Dir Assoc 2007; 8:322-7.

11. Vanasse GJ, Berliner N. Anemia in the elderly patients: an emerging problem for the $21^{\text {st }}$ century. Hematology Am Soc Hematol Educ Program 2010; 2010:271-5.

12. Tettamanti M, Lucca U, Gandini F, Recchia A, Mosconi P, Apolone G, et al. Prevalence, incidence and types of mild anemia in the elderly: the "Health and Anemia" population-based study. Haematologica 2010; 95:1849-56.

13. Barbosa DL, Arruda HKG, Diniz AS. Prevalência e caracterização da anemia em idosos do Programa de Saúde da Família. Rev Bras Hematol Hemoter 2006; 28:288-92. 
14. Schaan MDA, Schwanke CHA, Bauer M, Luz C, Cruz IM. Hematological and nutritional parameters in apparently healthy elderly individuals. Rev Bras Hematol Hemoter 2007; 29:136-43.

15. Silva CLA. Fatores associados ao estado nutricional e ao nível de hemoglobina em idosos: Programa de Saúde da Família, Viçosa-MG [Dissertação de Mestrado]. Viçosa: Universidade Federal de Viçosa; 2008.

16. Santos IS, Scazufca M, Lotufo PA, Menezes PR, Benseñor IM. Anemia and dementia among the elderly: The São Paulo Ageing \& Health Study. Int Psychogeriatr 2012; 24:74-81.

17. Silva CLA, Lima-Costa MF, Firmo JOA, Peixoto SV. Nível de hemoglobina entre idosos e sua associação com indicadores do estado nutricional e uso de serviços de saúde: Projeto Bambuí. Cad Saúde Publica 2012; 28:2085-94.

18. Zakai NA, Katz R, Hirsch C, Shlipak MG, Chaves PHM, Newman AB, et al. A prospective study of anemia status, hemoglobin concentration, and mortality in an elderly cohort. Arch Intern Med 2005; 165:2214-20.

19. Riva E, Tettamanti M, Mosconi P, Apolone G, Gandini F, Nobili A, et al. Association of mild anemia with hospitalization and mortality in the elderly: the Health and Anemia population-based study. Haematologica 2009; 94:22-8.

20. Denny SD, Kuchibhatla MN, Cohen HJ. Impact of anemia on mortality, cognition and function in community-dwelling elderly. Am J Med 2006; 119:327-34.

21. Penninx BWJH, Pahor M, Woodman RC, Guralnik JM. Anemia in old age is associated with increased mortality and hospitalization. J Gerontol A Biol Sci Med Sci 2006; 61:474-9.

22. Dong X, de Leon CM, Artz A, Tang Y, Shah R, Evans D. A population based study if hemoglobin, race and mortality in elderly persons. J Gerontol A Biol Sci Med Sci 2008; 63:873-8.

23. Patel KV, Harris TB, Faulhaber M, Angleman SB, Connelly S, Bauer DC, et al. Racial variation in the relationship of anemia with mortality and mobility disability among older adults. Blood 2007; 109:4663-70.

24. Lima-Costa MF, Firmo JOA, Uchoa E. Cohort profile: The Bambuí (Brazil) Cohort Study of Ageing. Int J Epidemiol 2011; 40:862-7.

25. Lima-Costa MF, Uchoa E, Guerra HL, Firmo JOA Vidigal PG, Barreto SM. The Bambuí health and ageing study (BHAS): methodological approach and preliminary results of a population-based cohort study of the elderly in Brazil. Rev Saúde Pública 2000; 34:126-35.
26. Lima-Costa MF, Firmo JOA, Uchoa E. The Bambuí Cohort Study of Aging: methodology and health profile of participants at baseline. Cad Saúde Pública 2011; 27 Suppl 3:S327-35.

27. World Health Organization. Iron deficiency anemia. Assessment, prevention and control. Geneva: World Health Organization; 2001.

28. World Health Organization. Physical status: the use and interpretation of anthropometry: reporty of a WHO committee. Geneva: World Health Organization; 1998.

29. Plan and operation of the Third National Health and Nutrition Examination Survey, 1988-94. Series 1: programs and collection procedures. Vital Health Stat 1 1994; (32):1-407.

30. Rose GA. The diagnosis of ischaemic heart pain and intermittent claudication in field surveys. Bull World Health Organ 1962; 27:645-58.

31. Castro-Costa E, Fuzikawa C, Ferri C, Uchoa E, Firmo J, Lima-Costa MF, et al. Dimensions underlying the mini-mental state examination in a sample with low-education levels: the Bambuí health and aging study. Am J Geriatr Psychiatry 2009; 17: 863-72.

32. Castro-Costa E, Fuzikawa C, Uchoa E, Firmo JOA, Lima-Costa MF. Norms for the Mini-Mental State Examination: adjustment of the cut-off point in population-based studies (evidences from the Bambuí health aging study). Arq Neuropsiquiatr 2008; 66:524-8.

33. Chalmers KA, Knuiman MW, Divitini ML, Bruce DG, Olynyk JK, Milward EA. Long-term mortality risks associated with mild anaemia in older persons: the Busselton Health Study. Age Ageing 2012; 41:759-64.

34. Lima e Costa MF, Barreto SM, Guerra HL, Firmo JOA, Uchôa E, Vidigal PG. Ageing with Trypanosoma cruzi infection in a community where the transmission has been interrupted: the Bambuí Health and Ageing Study (BHAS). Int J Epidemiol 2001; 30:887-93.

35. Lima-Costa MF, Cesar CC, Chor D, Proietti FA. Selfrated health compared with objectively measured health status as a tool for mortality risk screening in older adults: 10-year follow-up of the Bambuí Cohort Study of Aging. Am J Epidemiol 2012; 175:228-35.

36. Beghé C, Wilson A, Ershler WB. Prevalence and outcomes of anemia en geriatrics: a systematic review of literature. Am J Med 2004; 116 Suppl 7: S3-10.

Recebido em 27/Dez/2012

Versão final reapresentada em 15/Mai/2013

Aprovado em 23/Mai/2013 